\title{
Poster: AR-based Social Presence Enhancement in Video-chat Communication
}

\author{
Igor de Souza Almeida*, Marina Atsumi Oikawa*, Jordi Carres Polo*, Jun Miyazaki*, Mark Billinghurst**, Hirokazu Kato* \\ ${ }^{*}$ Nara Institute of Science and Technology ${ }^{* *}$ University of Canterbury
}

\begin{abstract}
Video-mediated communication systems attempt to provide users with a channel that could bring out the "feeling" of face-to-face communication. Among the many qualities these systems aim for, a high level of Social Presence is unquestionably a desirable one; however, little effort has been made to improve upon the user's perception of "presence". We propose an AR approach to enhance social presence for video-mediated systems by allowing one user to be present in the other user's video image. By using one extra camera on both sides, our system captures and merges the hand image from/to both sides resulting in an augmented experience where each user can "reach" to the other user's world. We conducted a preliminary pilot study with 10 participants coupled in 5 pairs in order to evaluate our system compared to the traditional video-chat setup. Tasks with varying equality of roles for the participants were performed. Results indicated that our system has higher degree of social presence compared to traditional video-chat systems. This conclusion was supported by the positive feedback from the subjects.
\end{abstract}

KEYWORDS: augmented reality, social presence, video-mediated communication.

INDEX TERMS: H.5.1 [Information Interfaces and Presentation]: Multimedia Information Systems-Artificial, augmented, and virtual realities_Evaluation/Methodology; H.4.3 [Communications Applications]: Computer conferencing, teleconferencing, and videoconferencing.

\section{INTRODUCTION}

Video mediated communication systems are widely spread and easily accessible in their "video-chat" form, reason why it is one of the most popular communication channels being used between remotely located people. Traditional video-chat systems rely primarily on the two-way video/audio feedback in order to cover the cues for an efficient human-human communication. However, taking into account that face-to-face communication is the optimal communication case, there are a number of natural non-verbal communication cues which help one to convey the message, including gaze direction, proximity behavior and pointing in space [1][2].

Researchers have since explored new concepts to offer a more complete experience. The idea of Shared Space is one of them. Shared space draws similarities with Collaborative Virtual Environments (CVE) in which participants and information share a common display space [3]. Even though it has been proven that using a dedicated shared space environment improves the results

\footnotetext{
igor-a@is.naist.jp

marina-o@is.naist.jp

jordi-p@slis.tsukuba.ac.jp

miyazaki@is.naist.jp

mark.billinghurst@canterbury.ac.nz

kato@is.naist.jp
}

of certain tasks [4][5], it does not address the effect of having an additional separated environment aside from the users' real world.

In HyperMirror [6], a shared scene is created by capturing the front view of the users on one side and merging the images of all users in one video image. Although the resulting image displays all users as if they were side-by-side in front of a mirror, a user trying to interact with another sideways has to keep the focus forward.

In this work, we intend to improve upon the traditional videochat setup which typically consists of two windows being presented to both ends: one to display the user's own video image and another one to show the remote person. By adding one extra camera focusing on a shared space with monochromatic background, a user can place his/her hand/s inside this space and then have them combined with the other user's face image. At the same time it simulates their coexistence, it stimulates different interaction patterns as previously reported by [7]. We advocate that this has the potential to enhance social presence.

Social presence has been defined as the salience of the partner in a mediated communication and the consequent salience of their interpersonal interactions [8]. However, [9] also distinctively points out that social presence has been shown to relate more to the user's perception of a medium's ability to provide salience of another as opposed to measuring the actual perceived salience of another person. For this work, social presence is targeted as the measure to perceive how much the presence of one user's video image into another user's video image can enhance sense of "being part of" each other's environment.

\section{HANDY SYSTEM}

In our proposed system, the main idea is to minimize the feeling of being geographically separated by allowing one user to be part of the video image of the other user. Figure 1 shows a comparison between the setup of a traditional video-chat system and our proposed system, referred to as HANDY. In the former, User B (in red background) has one camera capturing his face image which is displayed in the small window on top of User A's face image (in blue background) in the big window. Users are restricted to interact in their own environment.

In the latter, HANDY system, there is an extra camera focusing on the hand gestures alongside the camera in the traditional setup. The hand image is then sent and merged into the other user's face image. In Figure 1, it is possible to see the hand of User A merged with User B's face image in the small window and the hand of User B merged with User A's face image in the big window, as if they had crossed to the other side.

The shared space requires a training phase using the second camera (hand camera) using the first $n$ initial frames containing the monochrome background. The training step is necessary to allow the segmentation of foreground and background pixels from an incoming frame according to a threshold value $t$. After this segmentation, the background is subtracted and the image's parts of interest (the hands) are merged with the remote user's video image in real time. 

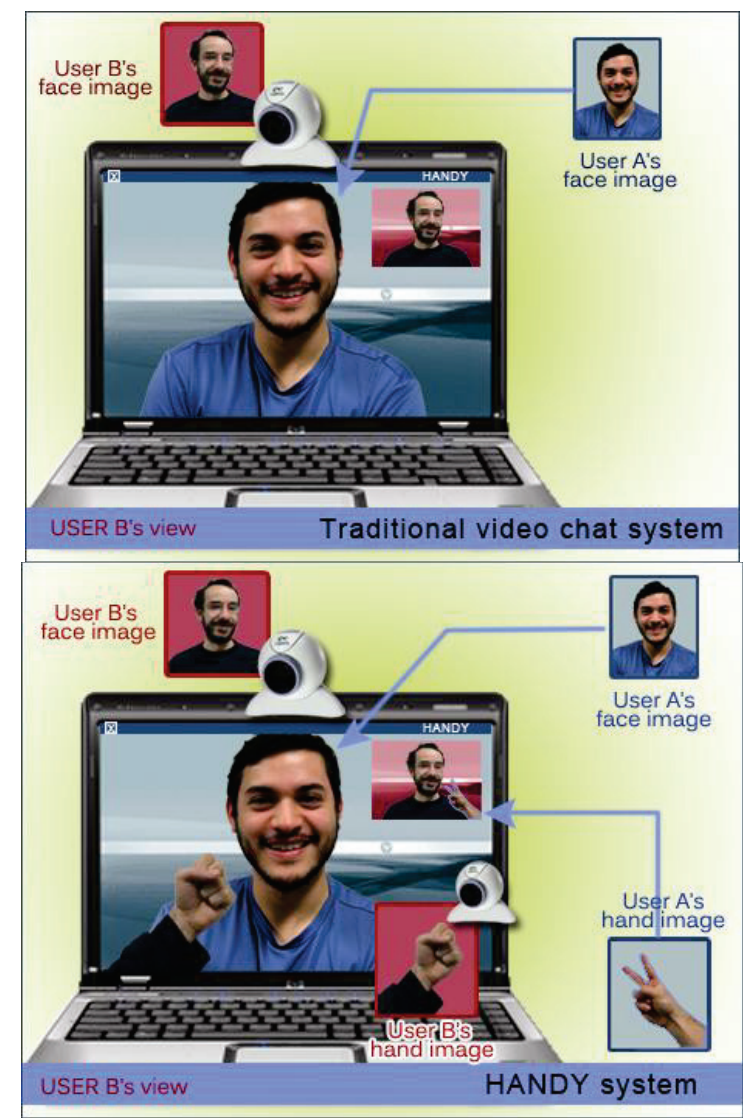

Figure 1. Conceptual images of the setup for a traditional video chat system (above) and HANDY system (below) considering user B's view.

The use of a simple technical solution made it affordable when applying it to video-chat system in contrast to more complex solutions such as pure Augmented Reality. In addition, it accelerated the prototyping process. The idea is to demonstrate the potential of the system by achieving equivalent visual results without the need to implement all the complexity involved in the pure AR solution. Nevertheless, the use of a high quality virtual image along with the proper $3 \mathrm{D}$ registration could potentially bring a more polished and highly interactive result.

\section{EVALUATION}

We designed a pilot user study comparing HANDY against the traditional video-chat system to evaluate the degree of Social Presence as well as Ease of Use, Enjoyment, Ease of Communication, Intuitiveness, Ease of understanding and Closeness. Co-presence was introduced as a measure of mutual perception. We had a total of 10 participants in the study comprising 8 Japanese students and 2 Foreign students, 23 to 35 years old (average 25.5), all male, all graduate students from the Interactive Media Design Lab. They performed in pairs throughout the experiments.

Overall, three tasks were performed by each pair: a Rock-paperscissors game; a Puzzle Matching where participants had to find the match for each puzzle; and an Origami Training task where one user taught another how to fold the paper into a predetermined shape.
Our hypotheses about the study's outcome were mainly targeting Social Presence and they can be described as follows:

H1 $1_{0}$ : HANDY On and HANDY Off generate a similar degree of measured social presence.

H1a: HANDY On generates a higher degree of measured social presence in comparison on HANDY Off.

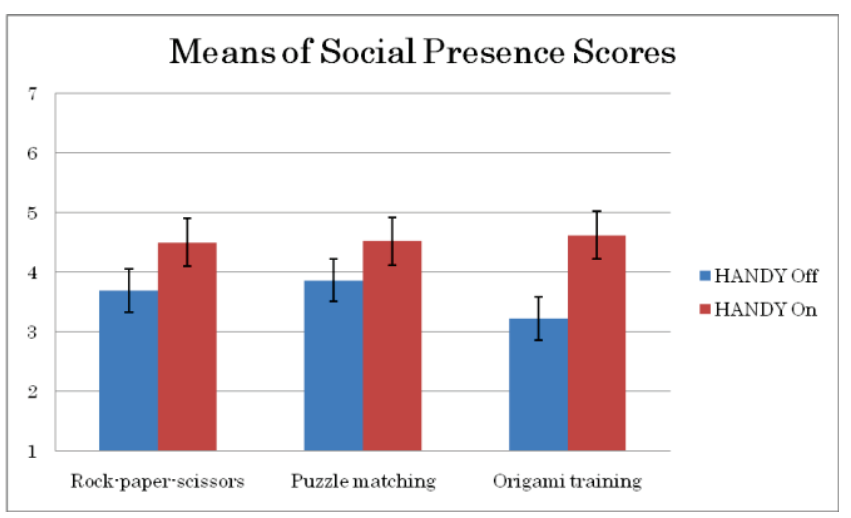

Figure 2. Social Presence average ratings and standard errors.

Despite the small number of samples, significant differences were found between conditions for all factors. Post-hoc comparisons showed that social presence was significantly higher in condition HANDY On $(\mathrm{Mean}=3.59, \mathrm{StDev}=1.14, \mathrm{p}<0.05)$ than HANDY Off $(\mathrm{Mean}=4.54, \mathrm{StDev}=1.27, \mathrm{p}<0.05)$. Figure 2 shows the means of social presence for each condition over the tasks.

\section{REFERENCES}

[1] Joerg Hauber. Understanding Remote Collaboration in Video Collaborative Virtual Environments. Master's Thesis, University of Canterbury, Christchurch, New Zealand, 2008.

[2] Carmen Edigo. Teleconferencing as a technology to support cooperative work: Its possibilities and limitations. In Intellectual Teamwork: Social and Technological Foundations of Cooperative Work. Hillsdale, NJ: Lawrence Erlbaum Associates, 1990.

[3] Steve Benford. Shared Spaces: Transportation, Artificiality, and Spatiality. In Proceedings of CSCW '96, pages 77-86, 1996.

[4] Judith S. Olson, J.S, Gary M. Olson and David K. Meader. What Mix of Video and Audio is Useful for Small Groups Doing Remote Real-time Design Work? In Proceedings of the SIGCHI '95, pages 362-368, 1995.

[5] Steve Whittaker, Geelhoed, E. and Robinson E. Shared workspaces: how do they work and when are they useful? In International Journal of Man-Machine Studies, volume 39, pages 813-842. Academic Press Ltd, 1993.

[6] Osamu Morikawa and Takanori Maesako. HyperMirror: toward pleasant-to-use video mediated communication system. In Proceedins of CSCW'98, pages 149-158. ACM Press, 1998.

[7] Charlotte N. Gunawardena. Social Presence Theory and Implications for Interaction and Collaborative Learning in Computer Conferences. In International Journal of Educational Telecommunications, volume 1, pages 147-166. Charlottesville, VA, AACE, 1995.

[8] John Short, Ederyn Williams and Bruce Christie. The social psychology of telecommunications. Wiley Ltd, London, UK, 1976.

[9] Eva-Lotta Sallnas. Effects of Communication Mode on Social Presence, Virtual Presence and Performance in Collaborative Virtual Environments. In Presence: Teleoperators and Virtual Environments, volume 14, pages 434-449. MIT Press, Cambridge, USA, 2005. 